\title{
Endoscopic Approach to the Internal Auditory Canal
}

\author{
Abdulrahman Dia*, Kevin M. O’Grady and Miriam I. Redeaf
}

\author{
University of Illinois at Chicago, USA
}

\begin{abstract}
Endoscopic approaches and techniques of otologic surgery are becoming more prevalent. The use of endoscopy in cholesteotoma, tympanoplasty, and other middle ear surgery is extensively documented [1,2]. Furthermore, the use of endoscopy to assist in established transcranial approaches to the internal auditory canal (IAC) and cerebellopontine angle (CPA) is becoming more widely accepted [3]. Retrosigmoid resections of CPA tumors using only endoscopic visualization have been described $[4,5]$.
\end{abstract}

Keywords: Endoscopy, internal auditory canal (IAC), cerebellopontine angle (CPA), otologic surgery.

\section{INTRODUCTION}

Established use of the transotic approach to the IAC has been to expand translabyrinthine approaches to resect large tumors or to reach the petrous apex [6,7]. To date, no direct exclusively endoscopic transotic approaches have been reported.

We describe an exclusively endoscopic approach through the cochlea and vestibule in order to section the auditory nerve, the vestibular nerves, or to remove small lateral IAC tumors. This approach is anatomically direct and requires less drilling than previous approaches.

\section{METHODS}

A fresh human cadaver head specimen was used. The specimen was stored in a laboratory refrigerator at $1-3^{\circ} \mathrm{C}$. Throughout the dissection, rigid Stryker $4 \mathrm{~mm}$ nasal endoscopes $\left(0^{\circ}\right.$ and $\left.30^{\circ}\right)$ were used for visualization. A Karl Storz micro-camera was used, with a $150 \mathrm{~W}$ light source, color monitor and high-definition video recorder system.

The preferred incision is designed to allow easier access to the external canal without interference from the cartilaginous canal, and to allow for oversewing the external canal at the end of the case, if desired. Specifically, an incision in the inferior half of the postauricular crease is carried around the attachment of the lobule (Fig. 1A). Skin and soft tissue planes are developed to allow for a pedicled pericranial flap to rotate under the lateral external canal, if desired (Fig. 1B). The bony external canal is then exposed, and the skin of the canal, the annulus, and the tympanic membrane are removed, revealing the middle ear space (Fig. 1C). A narrowed external boney canal can be widened and the ossicles are removed with care to preserve the chorda tympani (Fig. 1D).

The rigid endoscope is used to identify the promontory, Eustachian tube orifice, facial nerve, tensor tympani muscle,

*Address correspondence to this author at the University of Illinois at Chicago, 1855 West Taylor Street, Chicago, Illinois 60612, USA; Tel: 312996-6583; E-mail: Adia3@uic.edu cochleoform process, and open vestibule (Fig. 2-drawing). For illustration here only, the carotid artery is delicately skeletonized to demonstrate its location and its protection by the thick capsule of the promontory. In an actual patient, the carotid is not skeletonized.

Next, the basal and middle turns of the cochlea are carefully opened on their lateral surface to establish the orientation of the cochlea which is approximately $25^{\circ}$ off the horizontal. When viewed with an endoscope, the apex of the cochlea appears to be superior-laterally oriented. Once the orientation of the cochlea is established, the osseous spiral lamina, intrascalar septae and modiolus are shelled out to leave the fundus of the cochlea (Fig. 3A). The auditory nerve is seen with firm fibrous attachments around it.

With extreme care to stay inferior to the horizontal facial nerve, the oval window is opened inferiorly and anteriorly to expose the vestibule and the recesses containing the saccule and utricle (Fig. 3B). The party wall between the vestibule and the cochlea's capsule is removed, uniting the two chambers. Next the medial wall of the vestibule is opened to expose the inferior and superior vestibular nerves (Fig. 3C). Next, opening to the internal auditory canal is expanded to improve the view of the vestibular and facial nerves (Fig. 3D). Special note should be made that the internal auditory canal appears to course anteriorly from the cochlea and vestibule.

The facial nerve is identified directly medial to the superior vestibular nerve and is adherent to it. In cadaveric dissections, the identity of the facial nerve can be confirmed by further drilling of the superior portion of the cochlea to expose the labyrinthine portion. From the endoscopic transotic perspective, the internal auditory canal course anteriorly and the facial nerve is located superiorly in it (Fig. 4).

Fat is packed into the remaining walls of the vestibule and cochlea and down the Eustachian tube orifice. The mddle ear space is filled with fat. The soft tissue of the lateral external canal is closed in three layers, using the pedicled pericranial flap developed on opening. And the skin incision is closed. 


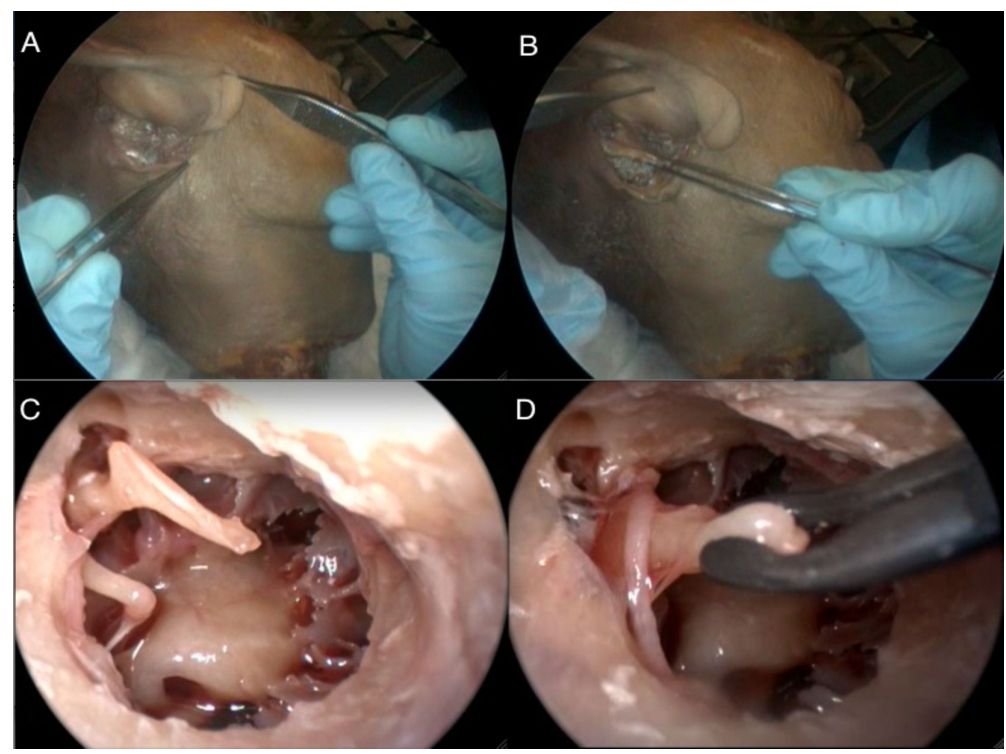

Fig. (1). Approach. A) Incision around the lobule attachment. B) Pericranial flap pedicled anteriorly on the external canal. C) Middle ear space after removal of the external canal skin. D) Removal of the incus. Malleus has already been removed.

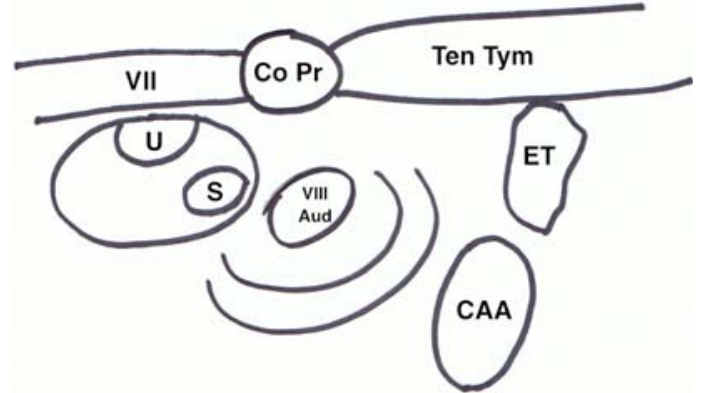

Fig. (2). Landmarks of the medial vestibule dissection. Tensor tympani (TenTym). Cochleoform process (CoPr). Facial nerve (VII). Utricle/saccule (U/S). Carotid artery (CAA)*. ET = Eustachian tube orifice. VIII Aud $=$ auditory nerve. ${ }^{*}$ Carotid not exposed during actual patient procedures.

\section{DISCUSSION}

Our exclusively endoscopic approach to the internal auditory canal (IAC) takes advantage of access through three open spaces in order to reach the IAC: the external auditory canal, middle ear space, and otic capsule. Thus, our method is anatomically direct, reduces drilling, and is minimally invasive. It also constitutes the transfer of endoscopic sinus surgery technology into otology.

In addition, our approach safely avoids the carotid artery. For purposes of illustration only in our dissection, we carefully identified the carotid artery anterior to the cochlea and delineated its path in the middle ear. (This step is not performed during an actual surgery.) Throughout our approach at no point was the carotid at risk as long as our

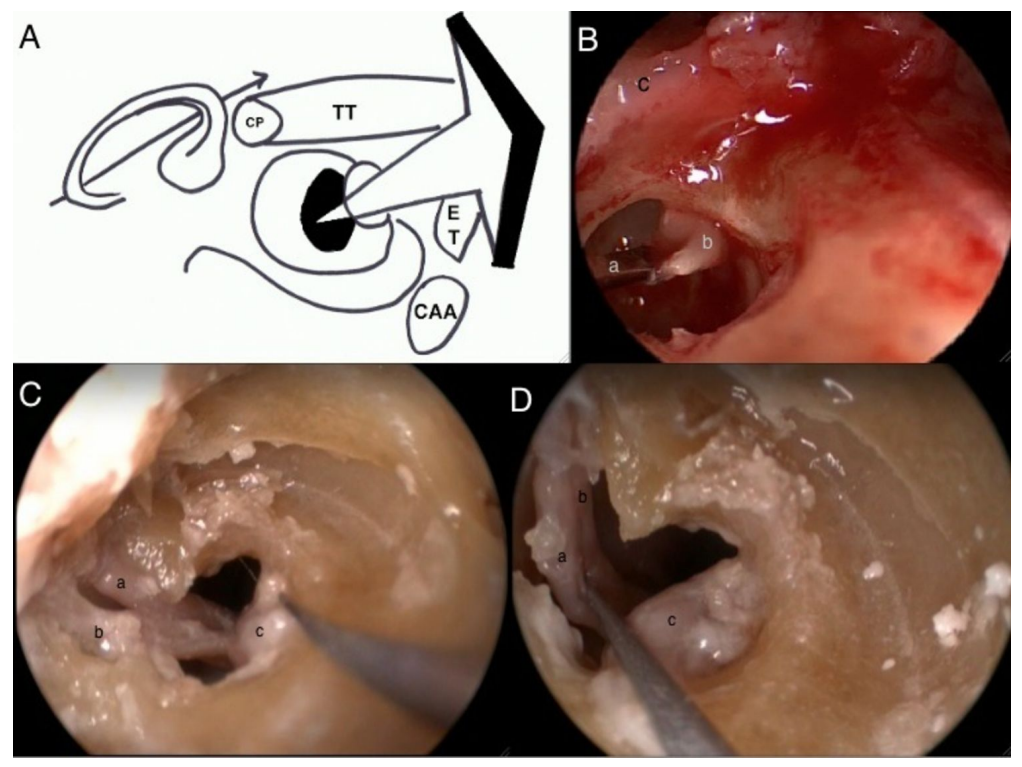

Fig. (3). A) Cochlear orientation. B) Saccule/utricle in the vestibule. a-instrument, b-utricule, c-facial nerve. C) Superior/inferior vestibular nerves within the IAC. a- superior nerve, b-inferior nerve, c-cochlear nerve. D) Inferior vestibular nerve has been sectioned. Superior vestibular nerve and facial nerve in the IAC. a- superior nerve, b-facial nerve, c-cochlear nerve. 


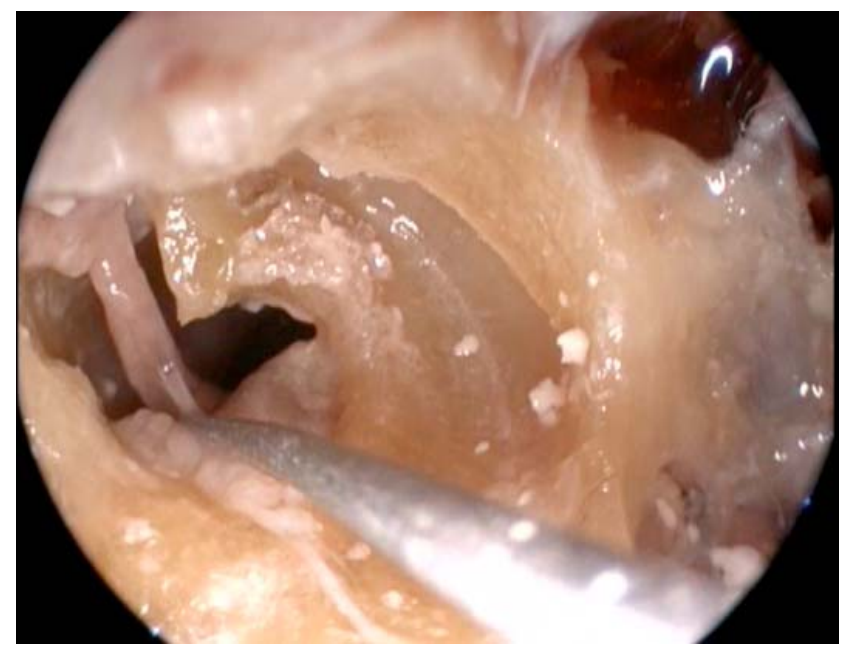

Fig. (4). Transotic view of the right internal auditory canal showing the lone facial nerve remaining. The superior basal turn of the cochlea has been drilled to expose the supra-cochlear route of the facial nerve. Note: the lumen of the internal auditory canal appears to course anteriorly.

drilling was confined to the walls of the cochlea. Thus, risk of rupturing the carotid artery is quite low.

This approach has specific utility. Convenient and direct access to the auditory, vestibular, and facial nerves makes this approach useful. Patients who complain of intractable dizziness with no acoustic function can benefit from vestibular nerve section via this direct method. Similarly, this approach can be applied to auditory nerve section in patients with uncontrollable tinnitus in a non-functioning ear. Lastly, patients with small lateral IAC tumors and poor hearing are ideal candidates for this anatomically direct procedure.

\section{ACKNOWLEDGEMENTS}

We would like to thank the University of Illinois at Chicago, with the department of Otolaryngology.

\section{REFERENCES}

[1] Tarabichi M. Transcanal endoscopic management of cholesteatoma. Otol Neurotol 2010; 31: 580-8.

[2] Migirov L, Shapira Y, Horowitz Z, Wolf M. Exclusive endoscopic ear surgery for acquired cholesteatoma: preliminary results. Otol Neurotol 2011; 32: 433-6.

[3] Lanman TH, Brackmann DE, Hitselberger WE, et al. Report of 190 consecutive cases of large acoustic tumors (vestibular schwannoma) removed via the translabyrinthine approach. J Neurosurg 1999; 90: 617-23.

[4] Sanna M, Russo A, Taibah A, Falcioni M, Agarwal M. Enlarged translabyrinthine approach for the management of large and giant acoustic neuromas: a report of 175 consecutive cases. Ann Otol Rhinol Laryngol 2004; 113: 319-28.

[5] Ben Ammar M, Piccirillo E, Topsakal V. Surgical Results and Technical Refinements in Translabyrinthine Excision of Vestibular Schwannomas: The Gruppo Otologico Experience. Neurosurgery 2012; 70: 1481-91.

[6] Shahinian HK, Ra Y. Fully endoscopic resections of vestibular schwannomas. Minim Invasive Neurosurgery 2011; 54(2): 61-7.

[7] Kabil MS, Shahinian HK. A series of 112 fully endoscopic resections of vestibular schwannomas. Minim Invasive Neurosurg 2006; 49: 362-8.

This is an open access article licensed under the terms of the Creative Commons Attribution Non-Commercial License (http: //creativecommons.org/licenses/by$\mathrm{nc} / 3.0 /$ ) which permits unrestricted, non-commercial use, distribution and reproduction in any medium, provided the work is properly cited. 